\title{
Time-resolved spectropolarimetric observations of polars WX LMi and BY Cam
}

\author{
D. Tutar Özdarcan, ${ }^{1,2 \star ~ P . ~ S . ~ S m i t h ~}{ }^{2 \star}$ and V. Keskin ${ }^{1 \star}$ \\ ${ }^{1}$ Department of Astronomy and Space Sciences, Faculty of Sciences, Ege University, Bornova Izmir 35100, Turkey \\ ${ }^{2}$ Steward Observatory, University of Arizona, Tucson, AZ 85721, USA
}

Accepted 2017 February 27. Received 2017 February 25; in original form 2016 July 30

\begin{abstract}
Time-series spectropolarimetric observations of polar WX LMi and asynchronous polar BY Cam are presented. Magnetic field properties, radial velocities and optical polarization are investigated via consecutive observations with good phase sampling during a single orbital cycle. Both systems are found to have a decentred dipole magnetic field configuration. One of the poles of WX LMi has a field strength of $49 \mathrm{MG}$, while the other pole may have possible field strengths of 69, 104 or 207 MG, depending on the harmonic numbers of the cyclotron humps observed in the circularly polarized spectrum. For BY Cam, a field strength of $168 \mathrm{MG}$ is found for one of the poles, while field strengths of 70, 160 or $212 \mathrm{MG}$ are possible for the other pole.
\end{abstract}

Key words: techniques: polarimetric-techniques: spectroscopic-stars: individual: BY Cam-stars: individual: WX LMi-stars: magnetic field-novae, cataclysmic variables.

\section{INTRODUCTION}

Magnetic cataclysmic variables (mCVs) are mass-transferring binary star systems with a magnetic white dwarf (WD) primary and a secondary red dwarf with a filled Roche lobe. The mass is transferred as a stream via the inner Lagrangian point (L1) from secondary to primary (Warner 1995). Among mCVs, polars form a subclass with very strong magnetic fields and synchronous rotation. The existence of a strong magnetic field does not allow formation of an accretion disc around the WD. Instead, the field forces charged particles (mostly electrons) in the mass transfer stream to move towards the magnetic poles of the WD. The strong magnetic field and accreting matter create magnetized plasma conditions in the vicinity of the magnetic poles of the WD. In such an environment, free electrons are forced to follow orbital paths that are perpendicular to the magnetic field lines. This movement of non-relativistic electrons produces circularly polarized cyclotron radiation in the emission region identified as the post-shock region within the accretion stream (Silva, Rodrigues \& Costa 2011) and is the source of the observed continuum polarization in the optical spectra of polars (Ingham, Brecher \& Wasserman 1976; Cropper 1990; Warner 1995).

WX LMi and BY Cam are two interesting systems that show cyclotron radiation in their optical continua and high circularly polarized emission. The polar nature of WX LMi was reported by Reimers, Hagen \& Hopp (1999), while the same classification was made for BY Cam by Remillard et al. (1986). WX LMi is clas-

\footnotetext{
^E-mail: demettutar@gmail.com (DTO); psmith@as.arizona.edu (PSS);
} varol.keskin@ege.edu.tr (VK) sified as a low accretion rate polar (LARP: Schwarz, Schwope \& Staude 2001). Schwope et al. (2002) mention that changes in accretion rates of polars occur without any obvious observed trigger or periodicity. They drew attention to the fact that most well-studied polars exhibit high and low states of accretion and identified a few systems that are observed in a persistent low state (LARPs), with accretion rates of $10^{-13} \mathrm{M}_{\odot} \mathrm{yr}^{-1}$. This accretion rate is about $10^{3}$ times smaller than that of a typical polar. In a later study, Vogel, Schwope \& Gänsicke (2007) classified WX LMi as pre-polar, i.e. the $\mathrm{mCV}$ has a secondary star underfilling its Roche lobe (Schmidt et al. 2005). In fact, the accretion rate of LARPs is consistent with the mass-loss rate of the secondary due to cool star winds, without Roche-lobe accretion. Webbink \& Wickramasinghe (2005) concluded that the strong magnetic field of a WD captures matter in the wind from the secondary and directs it to the magnetic poles of the WD. Since the mass accretion in LARPs is not due to Roche-lobe overflow, which is a defining criterion for a CV, Schwope et al. (2009) suggest that they are pre-cataclysmic binaries, or more precisely pre-polars. Schmidt et al. (2005) discussed the evolutionary state of CVs in the context of the magnetic field strength of the WD and the mass of the secondary component. They suggested that synchronization and accretion due to Roche-lobe overflow depend on the magnetic field strength of the WD and the size of the secondary component in pre-CVs. If a system has a modest magnetic field strength and a more massive secondary, it may evolve into an intermediate polar (IP), which would have an accretion disc around the WD and a spin period $\sim 20$ per cent shorter than the orbital period (Warner 1995). In the case in which the system has a strong magnetic field with a massive secondary, it may eventually become a polar. If the magnetic field is high enough to capture the matter in cool star winds ejected from a low-mass secondary star, 
synchronization may occur before the secondary fills its Roche lobe (Schmidt et al. 2005). With this evolutionary scenario, it is possible that pre-polars can be observed in an asynchronous state (Schmidt et al. 2005). In this context, Schwarz et al. (2001) emphasized the probability of asynchronization in WX LMi to explain the inferred magnetic field structure of the WD.

Reimers et al. (1999) found the orbital period of WX LMi to be $0.11638 \mathrm{~d}$ from photometry and spectroscopy. Schwarz et al. (2001) and Vogel et al. (2007) calculated orbital and spin periods by analysing spectroscopic and long-term photometric data. Vogel et al. (2007) suggested that the system is a synchronized rotator, with an orbital period derived from shifts of the hydrogen emission lines and sodium absorption lines attributed to the secondary component. The absorption lines in the observed spectrum must be from the secondary component in the system; the emission lines, however, can be from either the secondary or mass accretion in system, or both. For example, Mason, Liebert \& Schmidt (1989) describe the source of hydrogen and helium emission lines for the polar system BY Cam. They explain that strong Balmer lines can be categorized into four components: broad, narrow, high-velocity and asymmetric, depending on the origin of the emission. They attribute the broad component to the accretion stream above the accreting pole of the WD and claim that the narrow component originates near or on the secondary star. The high-velocity component arises from emitting material that falls faster than the bulk of the accretion stream and the asymmetric component indicates two-pole accretion separated in phase by 0.5 . Mason et al. (1989) emphasize that the blending of these emission components might be the source of the deviation from the expected sinusoidal orbital behaviour of the observed radial velocity curves.

In the case of BY Cam, Remillard et al. (1986) made the first orbital period estimation of $0.129 \pm 0.008 \mathrm{~d}$. A more precise period of $0.137120 \pm 0.000002 \mathrm{~d}$ was found by Honeycutt \& Kafka (2005) from long-term photometry. The synchronicity of BY Cam has been the subject of discussion in various articles, e.g. Mason et al. (1989) and Silber et al. (1991). Mason et al. (1998) showed evidence for asynchronous rotation of BY Cam of the order of $\sim 1$ per cent.

Patterson et al. (1995) considered asynchronous polars as a classification of BY Cam stars and they suggested episodic departures from synchronism for these systems. They proposed that a nova explosion may be the event that breaks the synchronization in these systems. Stockman, Schmidt \& Lamb (1988) observed asynchronous rotation in V1500 Cyg, a close binary system with a magnetic white dwarf component, and proposed that synchronicity was broken by its nova outburst in 1975 . They also identified the system as a polar (AM Her), suggesting that it was a synchronous rotator before the nova outburst. Later, Schmidt \& Stockman (1991) proposed that asynchronicity might be temporary in polars and that synchronous rotation could be re-established in a few hundred years. They concluded that AM Her systems, which have undergone a nova outburst and lost their synchronous rotation, would be synchronized again before the next nova outburst and that the time-scale for re-synchronization could be calculated via period variations determined from long-term observations. In the case of WX LMi and BY Cam, there have been no recorded nova explosions so far that could be used to test the idea proposed by Schmidt \& Stockman (1991). Schmidt \& Stockman (1991) also discussed the validity of this scenario from four years of photopolarimetric and photometric monitoring of the asynchronous polar BY Cam (Mason et al. 1989), but did not find any change in the rotation period. Very recently, Harrison \& Campbell (2016) announced that V1500 Cyg is synchronized again. This unexpected and early synchronization of the system is attributed to either an underestimated WD spin-down rate or an effect related to the high magnetic field strength.

Other photopolarimetric observations of BY Cam were performed by Mason, Liebert \& Schmidt (1987) and Piirola et al. (1994). Mason et al. (1987) found a modulation of circular polarization in BY Cam between 0 and 10 per cent over an orbital period and they drew attention to a change in the sign of the circular polarization from positive to negative within a period of about 20 orbital cycles. Piirola et al. (1994) reported +12.5 per cent positive circular polarization from $U B V R I$ measurements and calculated a magnetic field strength of $\sim 40 \mathrm{MG}$ for the $I$ band. Reimers et al. (1999) suggest that WX LMi has two accretion spots with magnetic field strengths of 60 and $68 \mathrm{MG}$ based on optical and X-ray spectroscopy.

Although photometric and spectroscopic observations of BY Cam and WX LMi exist in the literature, no optical spectropolarimetric observations have been published to date. In this study, we present the first time-resolved optical spectropolarimetry of these systems. Our observations cover a single orbital cycle with good phase sampling and enable us to calculate the radial velocities of the Balmer emission lines and construct the orbital parameters. Since the degree of circular polarization observed depends on the angle between the magnetic field axis and our line of sight (Warner 1995), tracing the strongest circular polarization feature is a suitable method to analyse the spin of the WD and also determine the number of accretion spots visible. In addition, we determine the location of cyclotron emission humps in the circularly polarized spectra and thereby estimate the magnetic field strength and configurations in these systems.

In the next section, we give a brief summary of observations and data reduction. In Section 3, we focus on radial velocities, circular polarization and magnetic field strength of the systems. We discuss and summarize our results in the last section.

\section{OBSERVATIONS AND DATA REDUCTION}

Observations were obtained using the 6.5-m Multiple Mirror Telescope (MMT) on Mount Hopkins, Arizona (AZ) and the 1.54-m Kuiper Telescope on Mount Bigelow, AZ. For all observations, the CCD spectropolarimeter SPOL was used (Schmidt, Stockman \& Smith 1992a). The polarimeter has a dual-beam design that incorporates rotating semi-achromatic wave plates and a full-aperture Wollaston prism. The CCD detector is a $1200 \times 800$ pixel $^{2}$ SITe CCD with quantum efficiency peaking at around 90 per cent at $\sim 6500 \AA$. A 964 line $\mathrm{mm}^{-1}$ diffraction grating spanning $\lambda \lambda 4090-7180 \AA$ in first order with a spectral resolution of 8-10 $\AA$ was used at the MMT, while a 600 line $\mathrm{mm}^{-1}$ grating yielding a resolution of $\sim 15 \AA$ was employed at the Kuiper telescope. The latter instrumental set-up provides a wavelength range of 3900-8400 A, but the ensuing analysis of the data is limited to 4200-7200 $\AA$, given the low signal-tonoise ratio of these data at the blue end of the spectrum and spectral order overlap issues at $\lambda>7600 \AA$. We summarize the observational parameters in Table 1 .

All observations were made using a sequence that rotates a $\lambda / 4$ wave plate to four position angles $90^{\circ}$ apart from each other. The $\mathrm{CCD}$ is read out twice during the sequence, with the first image sampling the two polarized beams from the Wollaston prism at wave-plate positions that are $180^{\circ}$ apart. The second image then samples the beams in the two wave-plate positions that are orthogonal to those for the first image. Exposure times listed in Table 1 are the total times needed for each measurement. The first line of Table 1 also gives the number of consecutive observations made. Each set of measurements cover the entire range of orbital periods 
Table 1. Observations with SPOL.

\begin{tabular}{lcc}
\hline & WX LMi & BY Cam \\
\hline$N^{*} \times$ exp. time (s) & $24 \times 240$ & $18 \times 600$ \\
UTC observation date & $2015 \mathrm{Apr} 28$ & 2014 Nov 29 \\
UTC start time & $06: 25: 24$ & $07: 32: 35$ \\
UTC end time & $08: 27: 04$ & $10: 45: 45$ \\
Slit width & $2.8 ; 44^{\prime \prime} 7$ & 4.1 \\
Telescope & MMT & Kuiper \\
\hline${ }^{*} N$ denotes the number of observations.
\end{tabular}

of the polars. Data reduction was carried out using standard IRAF ${ }^{1}$ (Tody 1986, 1993) packages and specific reduction scripts written for the SPOL instrument (see e.g. Schmidt et al. 1992b). The stars $\mathrm{BD}+28^{\circ} 4211$ (sdOp), G191 B2B (DAO) and HZ 44 (sdO) were observed as spectrophotometric calibration stars for both sets of observations. We present optical flux and circular polarization spectra of WX LMi and BY Cam in Figs 1 and 2, respectively.

\section{ANALYSIS}

\subsection{Time-resolved optical spectra and circular polarization}

Fig. 1 shows variations in the cyclotron continuum and intensity of Balmer emission lines throughout the orbital motion of WX LMi. The contribution of the late-type secondary component can be seen in the red portion of the optical spectra. Both optical flux and circularly polarized spectra exhibit similar cyclotron structures in the same wavelength ranges. We observe a maximum polarization at $\sim 5200 \AA$ from a cyclotron harmonic ('hump') that peaks at $\sim 50$ per cent negative circular polarization. When this polarized hump disappears with the orbital phase, two other humps appear at $\sim 4500$ and $\sim 5800 \AA$, reaching maxima of $\sim 33$ per cent positive circular polarization. This is evidence for mass accretion on to both poles of the WD of WX LMi.

The flux and polarization variations are not as dramatic in BY Cam as they are in WX LMi. In Fig. 2, we observe negative circular polarization up to -7 percent at the blue end of the spectrum during a portion of the orbital period and relatively weak positive circular polarization peaking at about 4 percent in the red during other orbital phases. These cyclotron features exhibit similar wide and shallow profiles in circularly polarized light. As with WX LMi, the opposite signs of circular polarization for cyclotron emission can be explained by mass accretion on to both magnetic poles of the WD component.

\subsection{Radial velocities and optical polarization}

With the availability of both optical flux and circularly polarized spectra, we are able to investigate the maximum polarization signal and wavelength shifts of Balmer emission lines during an orbital cycle. In Figs 3 and 4, we show the median spectra from all spectra obtained for WX LMi and BY Cam and the $\mathrm{H} \alpha$ emission-line profiles of the mCVs through an orbital cycle, respectively. Wavelength shifts can be seen in Fig. 4, as well as intensity variations. Since we do not observe any absorption lines in the observed wavelength range, we used the Balmer emission lines to calculate the orbital radial velocity for each observation. The first image of each target was

\footnotetext{
${ }^{1}$ The Image Reduction and Analysis Facility (IRAF) is distributed by the National Optical Astronomy Observatories, which are operated by the Association of Universities for Research in Astronomy, Inc., under cooperative agreement with the National Science Foundation.
}

used as a radial velocity template and then cross-correlated with the other images using the FXCOR package within IRAF. This determines the wavelength shifts, i.e. radial velocities, relative to the first image. After obtaining radial velocities, we apply the spectroscopic orbit solution by using a least-squares fit to the unweighted velocities. Adopting a circular orbit, we fit the orbital period, epoch (the time where maximum positive velocity is observed), semi-amplitude of the radial velocity curve and systemic velocity.

In addition to the radial velocities, we determine the maximum polarization percentage (positive and/or negative) for each image throughout the orbit and construct a time series of maximum polarization. Variations in time of the maximum observed polarization provide information on the spin period of the WD. However, the data do not cover more than a single orbit for both systems and this severely limits the accuracy of determination of WD spin periods. In Fig. 5, we plot measured radial velocities and best-fitting theoretical spectroscopic orbits, together with measured maximum circular polarization of each image and the best theoretical fits, as a function of time. A fairly good representation is achieved for the spectroscopic orbit of WX LMi. However, the radial velocities of BY Cam exhibit relatively large scatter around the theoretical orbit. Variable mass accretion could be a possible source of velocity scatter in polars (Mukai et al. 1986; Warner 1995) and possibly a factor for BY Cam. We make a preliminary period analysis of measured maximum circular polarizations using the PERIOD 04 (Lenz \& Breger 2005) software and find two dominant periods for both systems. By using these periods, we construct theoretical representations of the maximum polarization curves in Fig. 5. The main period indicates the spin period of the WD components, while the second period is half of the main period and has a $\sim 0.5$ phase difference with respect to the phase of the main period. This is evidence for two cyclotron radiation sources separated by $\sim 180^{\circ}$ in longitude, indicating a dipole magnetic field configuration in both systems.

\subsection{Magnetic field}

Variations in polarization and spectral flux depend on cyclotron radiation parameters in polars, such as magnetic field strength, electron number density and the angle between the magnetic field direction and our line of sight. However, due to the nature of cyclotron emission, the central wavelength of cyclotron humps can only be changed by the magnetic field strength. The central wavelengths of cyclotron features can thereby be used to find their associated harmonic numbers in the spectrum and the magnetic field strength can be calculated. The non-relativistic electron cyclotron radiation frequency, $\omega_{\text {ce }}$, for the fundamental frequency is obtained from classical electromagnetic theory (Ingham et al. 1976):

$\omega_{\mathrm{ce}(n)}=\frac{n|e| B}{2 \pi m_{\mathrm{e}} c}, \quad n=0,1,2, \ldots$,

where $m_{\mathrm{e}}$ is the mass of the electron, $e$ is the elementary charge, $c$ is the speed of light in vacuum, $B$ is the magnetic field strength and $n$ is the cyclotron harmonic number. Observed frequencies of harmonics increase with increasing harmonic number. The cyclotron frequency is equal to the difference between the frequencies of the two observed consecutive cyclotron humps in the spectrum. The following equation can be used to calculate the magnetic field strength using the peak wavelengths of the cyclotron humps instead of frequencies:

$\frac{|e| B}{2 \pi m_{\mathrm{e}} c^{2}}=\frac{1}{\lambda_{(n+1)}}-\frac{1}{\lambda_{(n)}}$. 


\section{WX LMi}

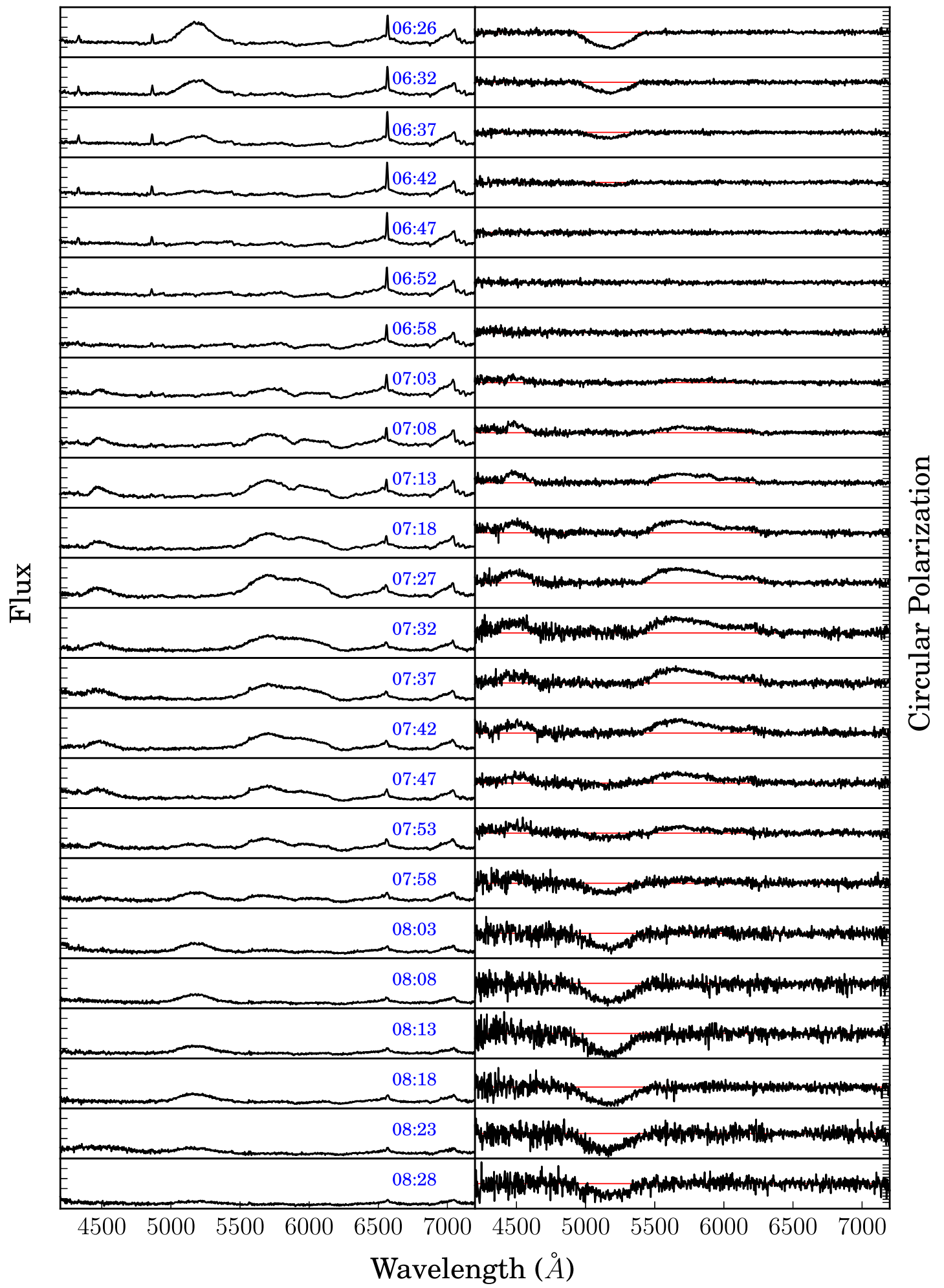

Figure 1. SPOL observations of WX LMi. Each row in the figure corresponds to a single simultaneous measurement of optical flux (left panels) and circular polarization (right panels) spectra. Flux density is given in units of erg cm $\mathrm{cm}^{-2} \mathrm{~s}^{-1} \AA^{-1}$. Ticks are placed from 0 to $15 \times 10^{-16} \mathrm{erg} \mathrm{cm}^{-2} \mathrm{~s}^{-1} \AA^{-1}$, with steps of $3 \times 10^{-16} \mathrm{erg} \mathrm{cm}^{-2} \mathrm{~s}^{-1} \AA^{-1}$. The scale for the circular polarization plots run from -60 per cent to 60 per cent and the red horizontal lines in the right panels represent 0 per cent circular polarization. The uT of the midpoint for each observation time is listed in the left panels. 


\section{BY Cam}

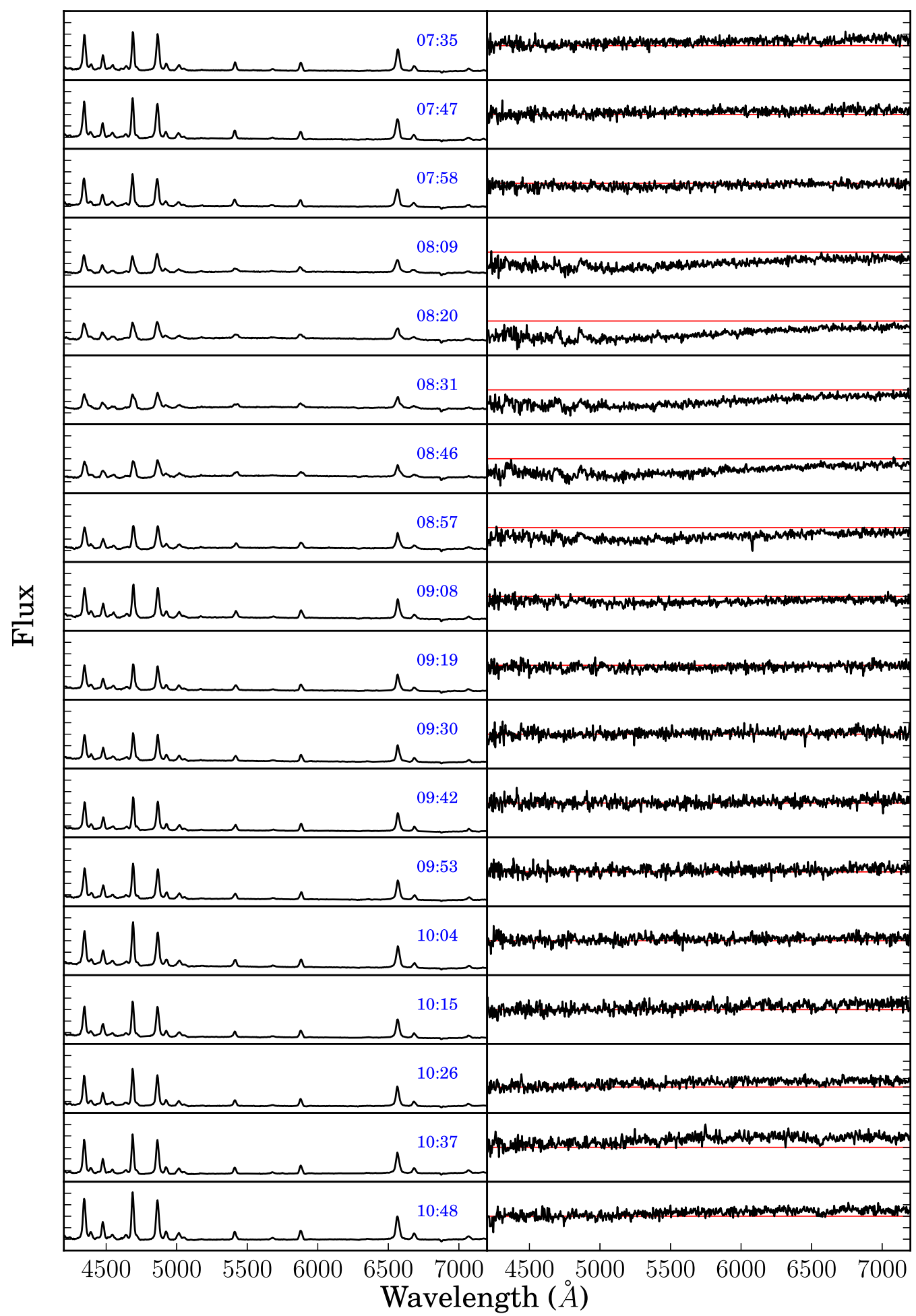

Figure 2. The same as Fig. 1, but for BY Cam. Ticks are placed from 0 to $18 \times 10^{-15} \mathrm{erg} \mathrm{cm}^{-2} \mathrm{~s}^{-1} \AA^{-1}$ in steps of $3 \times 10^{-15} \mathrm{erg} \mathrm{cm}^{-2} \mathrm{~s}^{-1} \AA^{-1}$ for flux. The scale for circular polarization ranges from -15 per cent to 15 per cent.

We plot optical and circular polarization spectra of WX LMi and BY Cam in Figs 6 and 7, respectively. For each system, the two orbital phases when the maximum positive and negative circular polarization occur are shown. We also mark the central wavelength of the cyclotron harmonics and identify the harmonics for WX LMi using equation (1). For WX LMi, two separate cyclotron radiation features are seen at 4484 and $5660 \AA$ in Fig. 6 (7:30 UT). The polarized features (maximum 33 per cent) are identified as the third and 

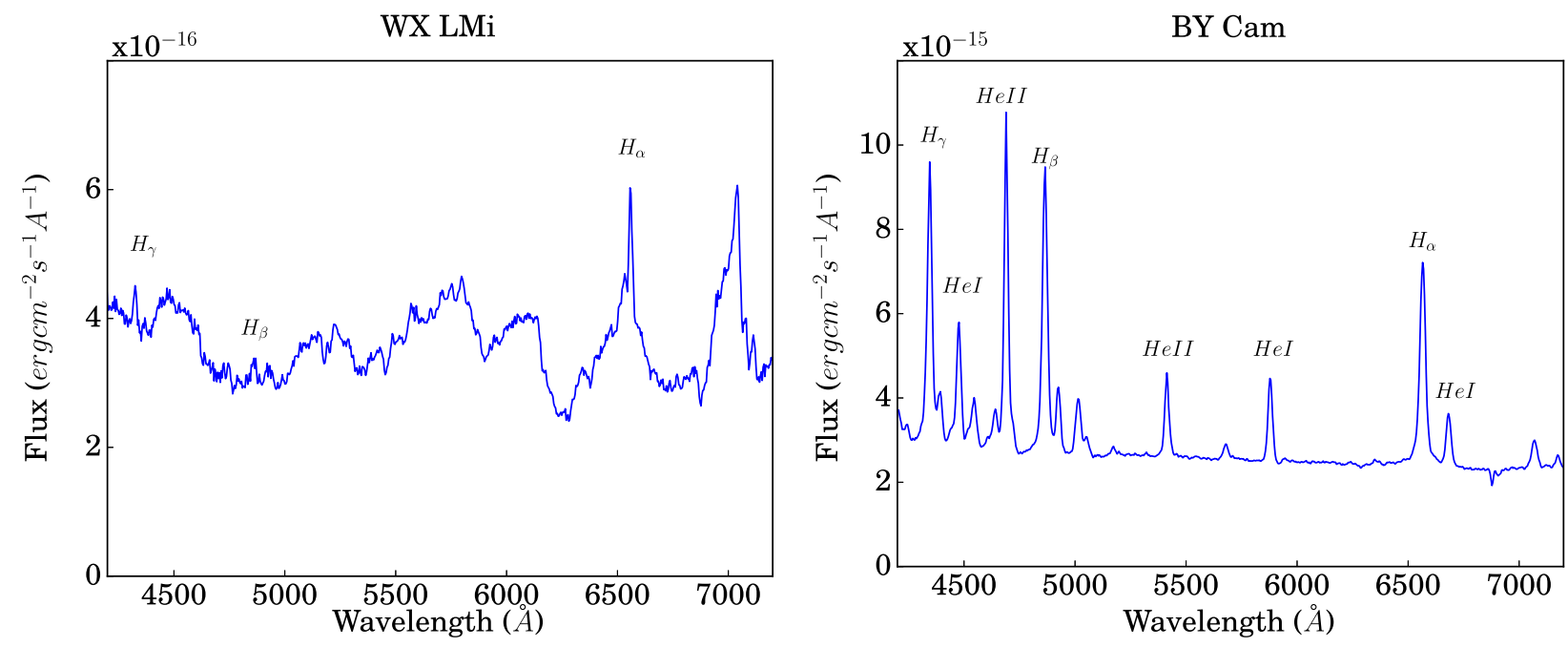

Figure 3. Calculated median spectra of WX LMi and BY Cam. Prominent hydrogen and helium emission lines are marked on each plot.
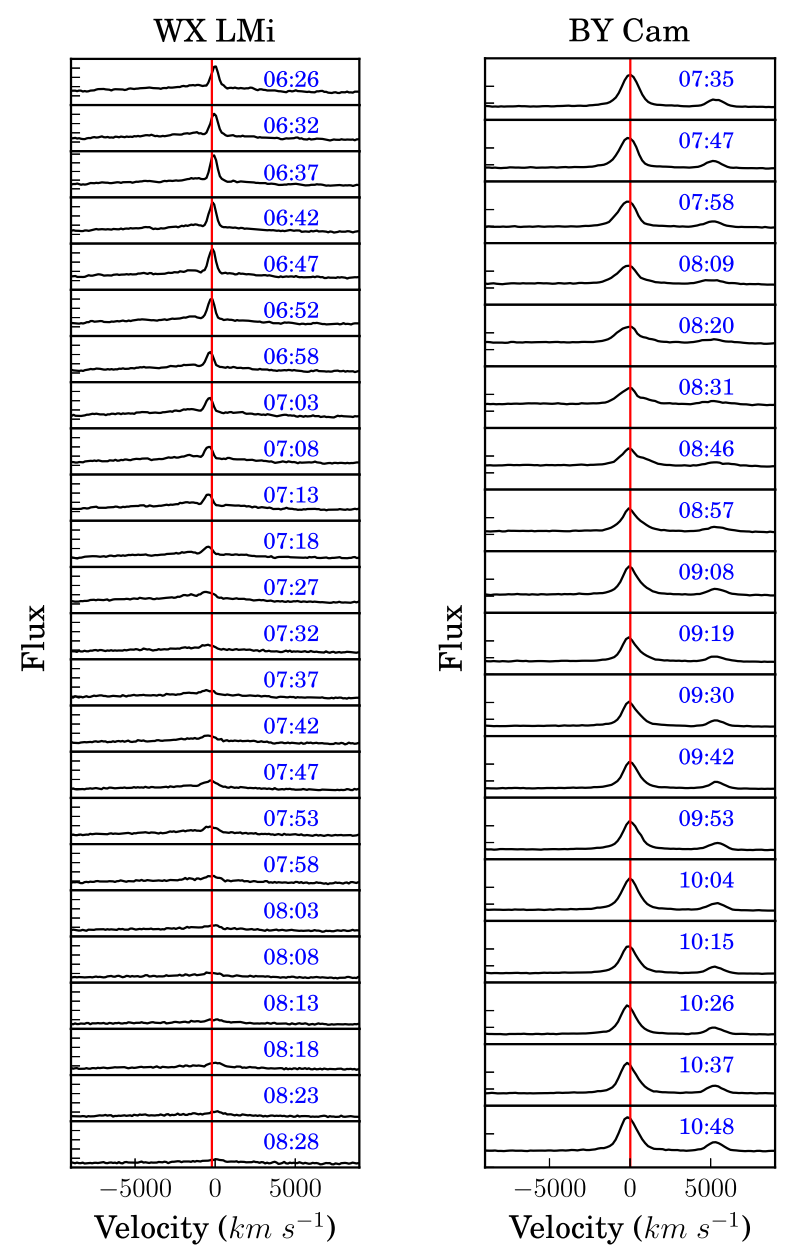

Figure 4. The $\mathrm{H} \alpha$ emission line oscillates about the systemic velocity because of orbital motion. BY Cam shows strong $\mathrm{H} \alpha$ at almost all orbital phases, while WX LMi exhibits similar strong emission for only a part of the orbit. As a reference, vertical red lines represent the systemic $\mathrm{H} \alpha$ radial velocity of both systems. Flux units are in $\mathrm{erg} \mathrm{cm}^{-2} \mathrm{~s}^{-1} \AA^{-1}$. Velocity shifts of emission lines of $\mathrm{H} \alpha$ are shown in the velocity domain. fourth harmonics, indicating a magnetic field strength of 49 MG. These structures are not visible by 8:11 uT, but another single cyclotron hump located at $5171 \AA$ becomes visible, with maximum 50 per cent negative polarization.

If a single hump is observed in the spectrum, it is not possible to assign a definitive harmonic number to the feature and determine the magnetic field strength directly. Assuming that the hump belongs to the fundamental frequency, we find an upper limit of the magnetic field strength via equation (1) as 207 MG. In order to estimate the lower limit of the magnetic field strength, we assume a harmonic number for the observed hump and calculate the harmonic numbers and the expected locations of the $(n+1)$ th and $(n-1)$ th harmonics via equation (1). The largest integer $n$ that puts the locations of adjacent harmonics out of the observed wavelength range yields the largest possible harmonic number for the observed feature. We find that the maximum harmonic number is 3 for WX $\mathrm{LMi}$, hence the lower limit of the magnetic field strength is $69 \mathrm{MG}$. If the observed hump belongs to the second harmonic, then the field strength is found as $104 \mathrm{MG}$. Two different sets of cyclotron features observed during the orbital period having different magnetic field strengths and opposite polarities indicate that the WD has a decentred dipole magnetic field configuration. This is not surprising for mCV systems in general, as suggested by Stift (1974) and Gerth \& Glagolevskij (2004). Vogel et al. (2007) have suggested an off-centred field configuration for WX LMi.

In the case of BY Cam, maximum polarizations of 4 (10:34 UT) and -7 per cent ( $8: 15$ UT) are seen in Fig. 7 . Each polarization feature may be from a single cyclotron hump that is very wide and shallow. Similar shallow cyclotron features were also observed and discussed for MR Ser (Wickramasinghe et al. 1991) and Wickramasinghe \& Meggitt (1985) explain such mild harmonic modulations in the spectra of mCVs as the result of high temperatures within the emission region. We fit a single Gaussian function to each hump to estimate the wavelength of the emission peak and adopt that wavelength as the location of the single cyclotron harmonic. Following the same approach as described for WX LMi, we calculate the largest cyclotron harmonic number and upper and lower limits on the magnetic field strength. In the left panels of Fig. 7, the harmonic number of the negatively polarized hump, which has a peak around $5060 \AA$, cannot be larger than 3 and this leads to a lower limit on the field strength of $70 \mathrm{MG}$. Assuming that the hump 
WX LMi
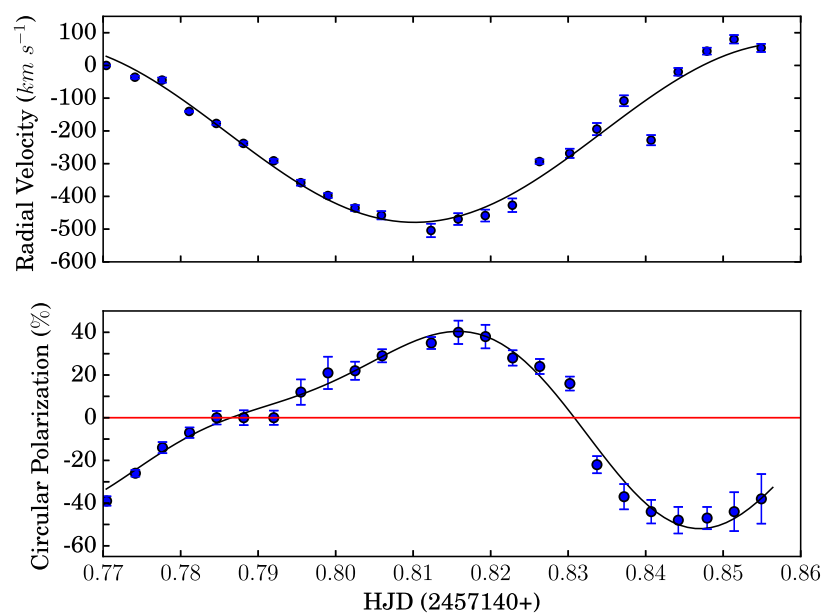

BY Cam
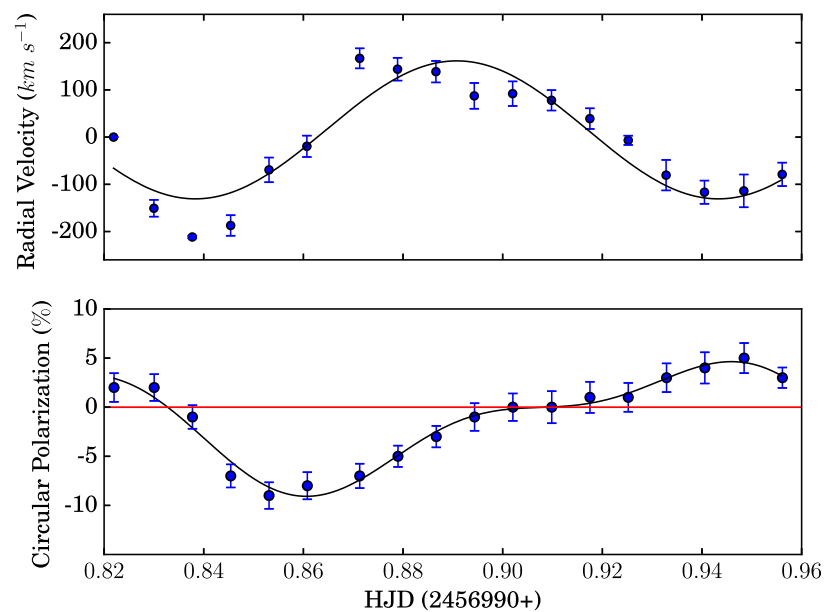

Figure 5. The measured radial velocity and maximum polarization are shown for all observations of WX LMi and BY Cam. In the upper panels, blue filled circles show measured radial velocities and continuous curves are theoretical representations of spectroscopic orbits. The orbital solution gives periods of $0.097 \pm 0.007 \mathrm{~d}$ for WX LMi and $0.105 \pm 0.006 \mathrm{~d}$ for BY Cam. In the lower panels, blue filled circles are maximum polarization percentages measured for each observation, while the curves display theoretical fits. Zero polarization is denoted by the horizontal red lines.
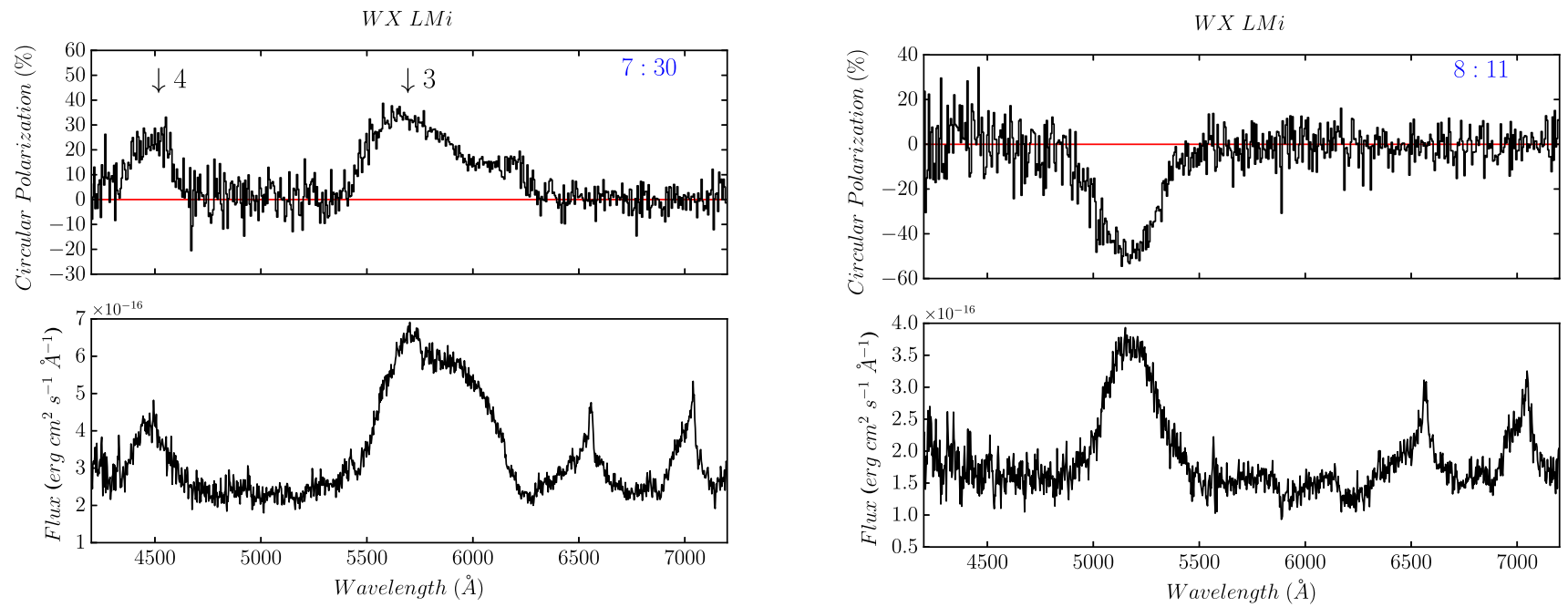

Figure 6. Simultaneously obtained optical circular polarization (upper panels) and flux (lower panels) spectra of WX LMi at two orbital phases. Cyclotron humps are identified by their harmonic number. The observing times are denoted in UT.

represents the fundamental frequency, we find an upper limit of the field strength of $212 \mathrm{MG}$. If the hump belongs to the second harmonic, then the field strength is $106 \mathrm{MG}$. The positively polarized hump has its maximum around $6353 \AA$, suggesting that it is at the fundamental cyclotron frequency and produced by a magnetic field of $168 \mathrm{MG}$.

\section{DISCUSSION AND SUMMARY}

In this study, we present time-resolved optical spectropolarimetric observations of WX LMi and BY Cam. Our observations allow us to determine radial velocities and circular polarization independently. Calculated orbital periods from the $\mathrm{H} \alpha$ emission lines are $0.097 \pm 0.007 \mathrm{~d}$ for WX LMi and $0.105 \pm 0.006 \mathrm{~d}$ for BY Cam. In addition, residuals from the spectroscopic orbital solution of BY Cam exhibit large scatter compared with the orbital solution of WX
LMi, which indicates additional larger velocity shifts due to the higher accretion rate in BY Cam.

Spin periods of the WDs are estimated by timing the appearance of the highest polarization features for each system. However, since our data only cover a single orbital cycle, these results are not definitive and further observations covering a few successive orbital cycles would be helpful in pinning down the parameters of these mCVs more accurately. Consequently, the calculated periods are not conclusive and not adequate for a discussion on the state of the synchronization of these systems.

WX LMi and BY Cam both exhibit cyclotron humps in their polarized spectra that vary along with the spin periods of WDs. We calculate magnetic field strengths from these cyclotron features and determine that both systems have magnetic dipole configurations. In the case of WX LMi, two opposite signed polarized cyclotron features occur at $\sim 0.5$ orbital phase apart from each other and the central wavelengths of these structures lead to different magnetic 

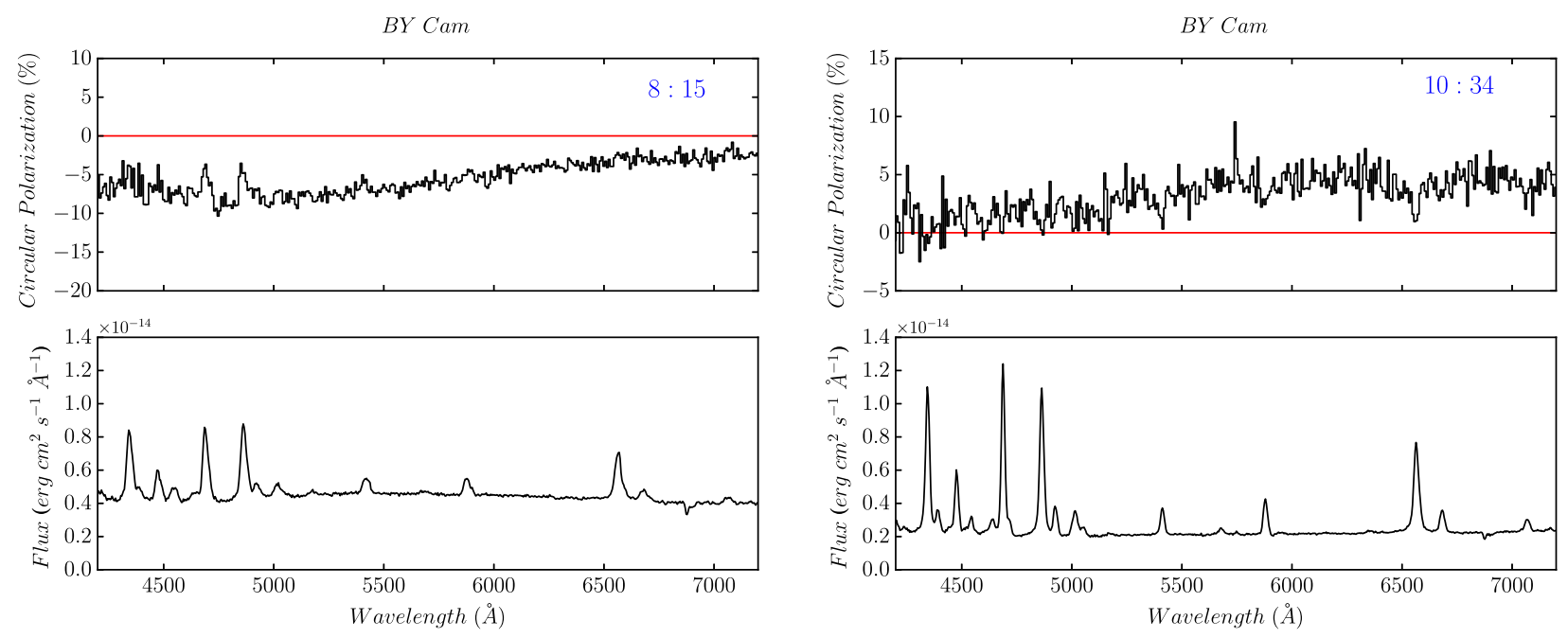

Figure 7. The same as Fig. 6, but for BY Cam.

field strengths. The observations indicate a magnetic field strength of $49 \mathrm{MG}$ for one pole and possible field strengths of 69,104 or $207 \mathrm{MG}$ for the other. These findings also show that the dipole magnetic field configuration is not centred on the centre of mass of the WD. For BY Cam, our observations show two broad cyclotron features with opposite polarities located $\sim 0.5$ apart in orbital phase. We find three possible field-strength values of 70, 106 or $212 \mathrm{MG}$ for one cyclotron radiating pole and $168 \mathrm{MG}$ for the other. Although BY Cam and WX LMi have similar magnetic field configurations, they exhibit very different optical flux and polarization spectra. The difference can be explained by different post-shock region parameters, such as magnetic field strength and accretion rate (Warner 1995). Our findings support this picture, i.e. BY Cam has weaker magnetic field strength and higher accretion rate than WX LMi.

Piirola et al. (1994) report that BY Cam shows either a negative or a positive polarization signal over an orbital cycle. Our observations show that both polarities are observed during an orbital cycle, indicating two different accretion regions separated by $180^{\circ}$ in longitude for the WD. These different behaviours over a time-scale of a few decades suggest significant long-term polarimetric variations in polars. This could possibly be related to a varying mass transfer rate in polars, as mentioned by Wickramasinghe \& Ferrario (2000), or pole switching as in the case of BY Cam, as discussed by Mason et al. (1998). Continued spectropolarimetric monitoring of WX LMi and BY Cam may be used to test temporary asynchronization states in polars, as well as tracing long-term changes in their polarization.

\section{ACKNOWLEDGEMENTS}

Observations of WX LMi reported were obtained at the MMT Observatory, a joint facility of the Smithsonian Institution and the University of Arizona. The authors thank Dr G. Grant Williams for providing an observation opportunity. D. Tutar Özdarcan thanks the Scientific and Technological Research Council of Turkey (TÜBITAK) for supporting this study through the 2214-A scholarship in 2014. D. Tutar Özdarcan also thanks the University of Arizona, Steward Observatory and MMT Observatory for their warm and kind hospitality during her observing runs and scientific visit in Tucson.

\section{REFERENCES}

Cropper M., 1990, Space Sci. Rev., 54, 195

Gerth E., Glagolevskij Y. V., 2004, in Zverko J., Žižnovský J., Adelman S. J., Weiss W. W., eds, Proc. IAU Symp. 224, The A-star Puzzle. p. 629

Harrison T. E., Campbell R. K., 2016, MNRAS, 459, 4161

Honeycutt R. K., Kafka S., 2005, MNRAS, 364, 917

Ingham W. H., Brecher K., Wasserman I., 1976, ApJ, 207, 518

Lenz P., Breger M., 2005, Commun. Asteroseismol., 146, 53

Mason P. A., Liebert J. W., Schmidt G. D., 1987, Inf. Bull. Variable Stars, 3104, 1

Mason P. A., Liebert J., Schmidt G. D., 1989, ApJ, 346, 941

Mason P. A., Ramsay G., Andronov I., Kolesnikov S., Shakhovskoy N., Pavlenko E., 1998, MNRAS, 295, 511

Mukai K. et al., 1986, MNRAS, 221, 839

Patterson J., Skillman D. R., Thorstensen J., Hellier C., 1995, PASP, 107, 307

Piirola V., Coyne G. V., Takalo S. J., Takalo L., Larsson S., Vilhu O., 1994, A\&A, 283, 163

Reimers D., Hagen H.-J., Hopp U., 1999, A\&A, 343, 157

Remillard R. A., Bradt H. V., McClintock J. E., Patterson J., Roberts W., Schwartz D. A., Tapia S., 1986, ApJ, 302, L11

Schmidt G. D., Stockman H. S., 1991, ApJ, 371, 749

Schmidt G. D., Stockman H. S., Smith P. S., 1992a, ApJ, 398, L57

Schmidt G. D., Bergeron P., Liebert J., Saffer R. A., 1992b, ApJ, 394, 603

Schmidt G. D. et al., 2005, ApJ, 630, 1037

Schwarz R., Schwope A. D., Staude A., 2001, A\&A, 374, 189

Schwope A. D., Brunner H., Hambaryan V., Schwarz R., 2002, in Gänsicke B. T., Beuermann K., Reinsch K., eds, ASP Conf. Ser. Vol. 261, The Physics of Cataclysmic Variables and Related Objects. Astron. Soc. Pac., San Francisco, p. 102

Schwope A. D., Nebot Gomez-Moran A., Schreiber M. R., Gänsicke B. T., 2009, A\&A, 500, 867

Silber A., Bradt H., Remillard R., Ishida M., Ohashi T., 1991, BAAS, 23, 880

Silva K. M. G., Rodrigues C. V., Costa J. E. R., 2011, preprint (arXiv:1101.5568)

Stift M. J., 1974, MNRAS, 169, 471

Stockman H. S., Schmidt G. D., Lamb D. Q., 1988, ApJ, 332, 282

Tody D., 1986, in Crawford D. L., ed., Proc. SPIE Vol. 627, IN: Instrumentation in Astronomy VI. SPIE, Bellingham, 733

Tody D., 1993, in Hanisch R. J., Brissenden R. J. V., Barnes J., eds, ASP Conf. Ser. Vol. 52, Astronomical Data Analysis Software and Systems II. Astron. Soc. Pac., San Francisco, 173 
Vogel J., Schwope A. D., Gänsicke B. T., 2007, A\&A, 464, 647

Warner B., 1995, Cataclysmic Variable Stars, Cambridge Astrophysics Series, 28. Cambridge Univ. Press, Cambridge

Webbink R. F., Wickramasinghe D. T., 2005, in Hameury J. M., Lasota J. P., eds, ASP Conf. Ser. Vol. 330, The Astrophysics of Cataclysmic Variables and Related Objects. Astron. Soc. Pac., San Francisco, p. 137 Wickramasinghe D. T., Ferrario L., 2000, PASP, 112, 873
Wickramasinghe D. T., Meggitt S. M. A., 1985, MNRAS, 214, 605

Wickramasinghe D. T., Cropper M., Mason K. O., Garlick M., 1991, MNRAS, 250, 692

This paper has been typeset from a $\mathrm{T}_{\mathrm{E}} \mathrm{X} / \mathrm{L} \mathrm{A} \mathrm{E} \mathrm{X}$ file prepared by the author. 